\title{
Qualidade de vida de pacientes em terapia renal substitutiva: uma análise da doença renal crônica e perfil populacional de risco
}

\author{
Quality of life of patients in renal therapy substitute: an analysis of chronic renal disease \\ and profile population of risk
}

\section{Calidad de vida de los pacientes sometidos a terapia renal sustitución: un análisis de la enfermedad renal crónica y el perfil población de riesgo}

Daniela Fernandes ${ }^{1 *}$, Tauane Letícia Pinto Zanelli ${ }^{1}$, Aline Scharr Rodrigues ${ }^{2}$, Mariana Paula Rodrigues $^{1}$, Jucilene Casati Lodi ${ }^{3}$, Tatiane Montelatto Marques ${ }^{1}$, Gislene dos Reis ${ }^{1}$, Luciane Ruiz Carmona Ferreira ${ }^{4}$, Clarice Santana Milagres ${ }^{1}$.

\section{RESUMO}

Objetivos: Avaliar a qualidade de vida de pacientes portadores de Doença Renal Crônica (DRC) em Terapia Renal Substitutiva (TRS), modalidade de hemodiálise, identificando os componentes e seus respectivos domínios comprometidos. Métodos: Estudo transversal, prospectivo, descritivo com abordagem quantitativa com portadores de DRC em TRS há no mínimo três meses, em uma instituição de saúde, interior do Estado de São Paulo. A coleta de dados foi realizada com 109 pacientes e utilizou-se os questionários sociodemográfico e de saúde; e o instrumento Avaliação da Qualidade de Vida (SF-36). Os dados foram analisados utilizando-se média, mediana e desvio padrão. Resultados: Dos sujeitos adultos participantes, $60 \%$ apresentaram um comprometimento na qualidade de vida relacionada as limitações físicas. Na estratificação os pacientes com condições clínicas comprometidas em início de tratamento sem acesso vascular definitivo, foi verificado comprometimento em variáveis físico e mental. Conclusão: Concluímos que a DRC causa impacto nos diferentes domínios dos compenentes físico e mental nos pacientes em tratamento hemodialítico. Tais comprometimentos podem implicar em significativo impacto, desempenho e percepção destes indivíduos sobre suas QV frente à doença e seu tratamento, um acompanhamento e monitoramento da equipe multidisciplinar.

Palavras-chave: Doença renal crônica, Grupos de risco, Qualidade de vida.

\begin{abstract}
Objectives: To evaluate the quality of life of patients with Chronic Kidney Disease (CKD) in Renal Replacement Therapy (RRT), hemodialysis modality, identifying the components and their respective domains involved. Methods: Cross-sectional, prospective, descriptive study with a quantitative approach with CKD patients on RRT for at least three months, in a health institution, in the interior of the State of São Paulo. Data collection was carried out with 109 patients and the sociodemographic and health questionnaires were used; and the Quality of Life Assessment instrument (SF-36). The data were analyzed using mean, median and standard deviation. Results: Of the participating adult subjects, $60 \%$ showed a compromise in quality of life related to physical limitations. In stratification patients with compromised clinical conditions at the beginning of treatment without definitive vascular access, impairment was verified in physical and mental variables. Conclusion: We conclude that CKD impacts the different domains of physical and mental components in patients undergoing hemodialysis. Such commitments can imply a significant impact, performance and perception of these individuals on their QOL in face of the disease and its treatment, a follow-up and monitoring of the multidisciplinary team.
\end{abstract}

Keywords: Chronic kidney disease, Risk groups, Quality of life.

\footnotetext{
${ }^{1}$ Centro Universitário da Fundação Hermínio Ometto (FHO), Araras - SP.

*E-mail: enfdfernandes@gmail.com

2 Universidade de São Paulo (USP), Ribeirão Preto - SP.

${ }^{3}$ Universidade Estadual de Campinas (UNICAMP), Piracicaba - SP.

${ }^{4}$ Universidade Estadual de Campinas (UNICAMP), Campinas - SP.
} 


\section{RESUMEN}

Objetivos: Evaluar la calidad de vida de los pacientes con enfermedad renal crónica (ERC) en terapia de reemplazo renal (TSR), modalidad de hemodiálisis, identificando los componentes y sus respectivos dominios involucrados. Métodos: Estudio descriptivo, prospectivo, transversal con enfoque cuantitativo con pacientes con ERC en TSR durante al menos tres meses, en una institución de salud, en el interior del Estado de São Paulo. La recolección de datos se realizó con 109 pacientes y se utilizaron los cuestionarios sociodemográficos y de salud; y el instrumento de evaluación de la calidad de vida (SF-36). Los datos se analizaron utilizando la media, la mediana y la desviación estándar. Resultados: De los sujetos adultos participantes, el 60\% mostró un compromiso en la calidad de vida relacionada con las limitaciones físicas. En la estratificación, los pacientes con afecciones clínicas comprometidas al comienzo del tratamiento sin acceso vascular definitivo, se verificó el deterioro de las variables físicas y mentales. Conclusión: Concluimos que la ERC afecta los diferentes dominios de los componentes físicos y mentales en pacientes sometidos a hemodiálisis. Dichos compromisos pueden implicar un impacto significativo, el rendimiento y la percepción de estos individuos sobre su calidad de vida frente a la enfermedad y su tratamiento, un seguimiento y monitoreo del equipo multidisciplinario.

Palabras clave: Enfermedad renal crónica, Grupos de riesgo, Calidad de vida.

\section{INTRODUÇÃO}

Considerada problema de saúde pública, devido crescente número de pacientes submetidos ao tratamento dialítico, a Doença Renal Crônica (DRC) é caracterizada pela perda progressiva e irreversível da função renal, onde a capacidade do corpo para manter o equilíbrio metabólico e hidroeletrolítico falha, resultando em uremia ou azotemia. Em seu estágio mais avançado é definido como Insuficiência Renal Crônica (IRC), onde os rins não podem manter sua funcionalidade (DIAS EC, et al., 2017).

No Brasil, segundo o censo brasileiro de diálise da Sociedade Brasileira de Nefrologia (SBN) referente a 2019, a distribuição de pacientes é de 93,2\% em hemodiálise (HD) e de apenas 6,8\% em diálise peritoneal (DP). Estima-se que o número de pacientes novos em diálise, por ano no Brasil, esteja em torno de 42.500, sendo que estes indicadores só tendem aumentar (SBN, 2020; NEVES, et al., 2020; THOMÉ, et al., 2019).

A DRC contribui para incapacidades e comprometimento na qualidade de vida destes indivíduos em Terapia Renal Substitutiva (TRS). A qualidade de vida acompanha os aspectos físico, mental e social dos indivíduos e pode impactar tanto positiva quanto negativamente na saúde do paciente renal crônico.

O acompanhamento da qualidade de vida destes portadores contribui no diagnóstico de fatores condicionantes que comprometem a saúde e recuperação, auxilia na implementação de ações terapêuticas e direciona o componente comprometido, extraindo assim melhores resultados para o tratamento proposto (GONÇALVES FA, et al., 2015).

Diante do exposto, o objetivo deste estudo é avaliar a qualidade de vida de pacientes portadores de DRC em TRS, na modalidade de HD, identificando os componentes e seus respectivos domínios comprometidos.

\section{MÉTODOS}

\section{Delineamento, local e participantes da pesquisa}

Pesquisa transversal, de caráter descritivo exploratório, conduzidos no serviço de nefrologia em uma instituição de saúde no interior do Estado de São Paulo, Brasil, em 2017. A população alvo foram indivíduos portadores de DRC em TRS, modalidade HD, cadastrados no serviço de nefrologia. De acordo com a rotina do serviço, os portadores se dividiam no turno matutino e vespertino, de segunda a sábado; e o noturno, as segundas, quartas e sextas-feiras, em três turnos.

Estavam cadastrados neste serviço cerca de 121 pacientes. Para o fechamento amostral foram incluídos: indivíduos portadores de DRC em HD, há pelo menos 3 meses, idade igual ou superior a 18 anos e concordassem em assinar o Termo de Consentimento Livre Esclarecido (TCLE). Os critérios de exclusão foram pacientes com déficit cognitivo ou incapazes de responder o questionário, que não respondessem a pesquisa em sua totalidade e se recusassem a participar. Da amostra total, duas pessoas se negaram, três tinham idade inferior a 18 anos e as demais encontravam hospitalizadas. Portanto, foram incluídos 109 participantes. 


\section{Instrumentos da Pesquisa}

A coleta de dados foi realizada por meio de entrevistas com questionário semiestruturado, aplicado diretamente ao paciente na instituição. A abordagem foi feita pelos pesquisadores, que apresentaram a pesquisa e solicitaram sua participação. Quando do aceite, após a assinatura do TCLE, os questionários foram expostos aos pacientes para o preenchimento durante a sessão de hemodiálise e entregues ao termino da sessão.

Após a conclusão da entrevista com o preenchimento dos questionários, decorreu-se a consulta nos arquivos e prontuário do paciente. Foram utilizados os seguintes instrumentos: questionário sociodemográfico, questionário de saúde e o SF-36 (Medical Outcomes Study 36).

\section{Questionário Sociodemográfico}

Questões fechadas com as seguintes variáveis: sexo, grupo étnico, estado civil, escolaridade, renda em salários mínimos, localização da moradia e número de pessoas residentes na moradia.

\section{Questionário de Saúde}

Intrumento com perguntas objetivas, realizadas diretamente aos pacientes, assim como dados clínicos obtidos através da busca em prontuário das informações foram coletados no questionário de saúde. As variáveis que o compunha foram: peso, altura, IMC, $\mathrm{Hb} / \mathrm{Ht}$ (Hemoglobina/Hematócrito), albumina, plano de saúde, internação em 2016, diagnóstico da doença de base, presença de DM, presença de HAS, sinais e sintomas prévios ao diagnóstico de DRC, tempo de realização de tratamento conservador em anos, tempo de realização de tratamento hemodialítico em, Kt/V , acesso para hemodiálise, fila no transplante renal e dificuldade alimentar/hídrica.

\section{SF 36 (Medical Outcomes Study 36)}

O SF 36 é um questionário genérico de avaliação da qualidade de vida, de fácil administração e compreensão. Consiste em um questionário multidimensional formado por 36 itens, agrupados em dois compomentes: componente saúde física (CSF) e o componente saúde mental (CSM).

O CSF apresenta quatro domínios: 1) capacidade funcional (desempenho das atividades diária); 2) aspectos físicos (impacto da saúde física no desempenho das atividades diárias e/ou profissionais); 3) dor (nível de dor e o impacto no desempenho das atividades diárias e/ou profissionais); e 4) estado geral de saúde (percepção subjetiva do estado geral de saúde).

O CSM apresenta mais quatro domínios: 1) vitalidade (percepção subjetiva do estado de saúde); 2) aspectos sociais (reflexo da condição de saúde física nas atividades sociais);3) aspectos emocionais (reflexo das condições emocionais no desempenho das atividades diárias e/ ou profissionais) e; 4) saúde mental (escala de humor e bem-estar).

Apresenta um escore final de 0 (zero) a 100 (obtido por meio de cálculo do Raw Scale), onde o zero corresponde ao pior estado geral de saúde e o 100 corresponde ao melhor estado de saúde (CICONELLI RM, 1997). Este questionário semiestruturado apresenta um delineamento validado para diversas propriedades reprodutibilidade, suscetibilidade à alterações necessárias, assim como validade.

\section{Autorizações e Procedimentos éticos}

Foi solicitado ao Serviço de Nefrologia do hospital do referido município a autorização para a coleta de dados. O projeto deste estudo foi encaminhado ao Comitê de Ética em Pesquisa (CEP) desta instituição, assim como para a Plataforma Brasil, conforme determinação da Resolução 196/96 do Conselho Nacional de Saúde para pesquisa envolvendo seres humanos, sendo aprovado com o parecer n097355/2016 e CAAE n 60181216.4.0000.5608.

\section{Análise dos Dados}

Para o armazenamento dos dados foi utilizado o software Office, Excel versão 2007 e para análise de dados foi utilizado o programa estatístico BioEstat 5.3. A normalidade da distribuição das variáveis quantitativas pesquisadas foi estimada usando o teste de Kolmogorov-Smirnov, enquanto a análise descritiva foi apresentada por medidas de tendência central, variabilidade adequadas e distribuição de frequências. 


\section{RESULTADOS}

Participaram do estudo 109 sujeitos. A idade idade média encontrada foi de 57,3 anos (DP $\pm 13,76)$. A amostra foi caracterizada por $60,6 \%$ dos pacientes com sexo masculino, $69,7 \%$ de etnia caucasiana e $60,5 \%$ casados. A escolaridade de até quatro anos de estudo e renda de até 3 salários mínimos estava presente em $71,6 \%$. A grande maioria, 97,2\%, reside em zona urbana, sendo $80,7 \%$ em casa própria e $59,6 \%$ moravam com 1 a 3 pessoas no domicílio (Tabela 1).

Tabela 1 - Variáveis sociodemográficas de pacientes. Limeira/SP, Brasil, 2017.

\begin{tabular}{ll}
\hline Variável & $\mathbf{N}(\%)$ \\
\hline Sexo & $60,6 \%$ \\
\hline Masculino & $34,4 \%$ \\
Feminino & $69,7 \%$ \\
\hline Grupo Étnico & $15,6 \%$ \\
\hline Caucasiano & $14,7 \%$ \\
Pardo & \\
Negro & $23,8 \%$ \\
\hline Estado Civil & $60,5 \%$ \\
\hline Solteiro & $8,2 \%$ \\
Casado & $7,5 \%$ \\
Divorciado & \\
Viúvo & $0,9 \%$ \\
\hline Escolaridade & $36,7 \%$ \\
\hline Analfabeto & $26,6 \%$ \\
Ens Fund 1 a 4 anos & $28,4 \%$ \\
Ens Fund 5 a anos & $7,4 \%$ \\
Ens Médio & \\
Ens Superior & $71,6 \%$ \\
\hline Renda (em salários mínimos) & $24,8 \%$ \\
\hline Até 3 SM & $0,9 \%$ \\
4 a 6 SM & $1,9 \%$ \\
7 a 9 SM & $0,9 \%$ \\
Acima de 12 SM & \\
\hline Localização da moradia & $97,2 \%$ \\
\hline Urbana & $2,8 \%$ \\
\hline Rural & \\
\hline Súmero de pessoas residentes na moradia & $9,2 \%$ \\
1 a 3 & $59,6 \%$ \\
4 ou mais & $31,2 \%$ \\
\hline Fonte: &
\end{tabular}

Fonte: FERNANDES D, et al., 2020.

Quanto aos dados relaciondos à saúde, 50,5\% afirmam ter plano de saúde e $45 \%$ tiveram ao menos uma internação no ano de 2016. A diabetes anterior à DRC foi relatada por $41,3 \%, 80,7 \%$ possuem hipertensão arterial e 70,6\% já apresentavam doença hipertensiva antes do problema renal.

O diagnóstico de base da doença renal mostrou $43,1 \%$ tem a doença com causa indefinida, seguida por nefropatia diabética. Sobre os sintomas apresentados previamente ao diagnóstico da doença renal, 30\% relataram edema e $21,1 \%$ descobriram a doença através dos exames alterados. Quanto ao tempo de tratamento da doença renal, $64,2 \%$ não tiveram período de tratamento conservador e o tempo em hemodiálise, até cinco anos é representado por $54,1 \%$ da amostra. O Kt/V maior ou igual à 1,2 foi encontrado em 47,7\%, 87,2\% usam fístula artériovenosa para a terapia, 37\% estão na fila para transplante renal e $55 \%$ relatavam dificuldade alimentar e hídrica (Tabela 2). 
Tabela 2 - Indicadores comorbidades e dados dialíticos da amostra estudada Limeira/SP, Brasil, 2017.

\begin{tabular}{|c|c|}
\hline Variável & $\mathbf{N}(\%)$ \\
\hline \multicolumn{2}{|l|}{ Diagnóstico da doença de base } \\
\hline IR Indefinida & $43,1 \%$ \\
\hline Nefropatia diabética & $18,4 \%$ \\
\hline Rim policístico & $10,1 \%$ \\
\hline Nefroesclerose hipertensiva & $7,3 \%$ \\
\hline Glomerupatias & $7,4 \%$ \\
\hline Demais doenças & $13,7 \%$ \\
\hline \multicolumn{2}{|c|}{ Sinais prévios aos dignostico de DRC } \\
\hline Edema & $30 \%$ \\
\hline Exames alterados & $21,1 \%$ \\
\hline Dispnéia & $9,2 \%$ \\
\hline Hipertensão arterial & $9,2 \%$ \\
\hline Outras & $38,5 \%$ \\
\hline \multicolumn{2}{|c|}{ Tempo que realizou tratamento conservador } \\
\hline Não teve & $64,2 \%$ \\
\hline$<1$ ano & $9,2 \%$ \\
\hline 1 a 2 anos & $12 \%$ \\
\hline 3 a 5 anos & $9,2 \%$ \\
\hline 6 a 10 anos & $2,7 \%$ \\
\hline$>10$ anos & $2,7 \%$ \\
\hline \multicolumn{2}{|c|}{ Período em tratamento hemodialítico (em anos) } \\
\hline$<1$ & $19,3 \%$ \\
\hline 1 a 2 & $28,4 \%$ \\
\hline 3 a 5 & $25,7 \%$ \\
\hline 6 a 10 & $21,1 \%$ \\
\hline 11 a 15 & $2,7 \%$ \\
\hline$>15$ anos & $2,7 \%$ \\
\hline \multicolumn{2}{|l|}{ Kt/V } \\
\hline$<1,2$ & $52,3 \%$ \\
\hline$\geq 1,2$ & $47,7 \%$ \\
\hline \multicolumn{2}{|l|}{ Acesso para hemodiálise } \\
\hline FAV & $87,2 \%$ \\
\hline Permcath & $7,3 \%$ \\
\hline CDL & $5,5 \%$ \\
\hline \multicolumn{2}{|l|}{ Fila para transplante renal } \\
\hline Sim & $37 \%$ \\
\hline Não & $63 \%$ \\
\hline \multicolumn{2}{|l|}{ Dificuldade alimentar/hídrica } \\
\hline Sim & $55 \%$ \\
\hline Não & $45 \%$ \\
\hline
\end{tabular}

Fonte: FERNANDES D, et al., 2020.

Os dados clínicos nos prontuários dos pacientes foram: peso $74,5 \mathrm{Kg}$ (DP $\pm 16,9)$, IMC $27 \mathrm{~kg} / \mathrm{m}^{2}$ (DP \pm 8,4 ), hemoatócrito 34,92 ( $\mathrm{DP} \pm 4,99$ ) e albumina $3,45 \mathrm{~g} / \mathrm{dL}$ ( $\mathrm{DP} \pm 0,45$ ). Na amostra, $58,7 \%$ dos sujeitos apresentaram IMC até $25 \mathrm{~kg} / \mathrm{m}^{2} ; 86,2 \%$ tinham hemoglobina maior que $13 \mathrm{~g} / \mathrm{dL}$ e $45 \%$ apresentaram $\mathrm{Kt} / \mathrm{V}$ maior que 1,4 .

$\mathrm{Na}$ avaliação das dimensões do SF-36 apresentados na Tabela 3 , foi verificado quanto à qualidade de vida dos sujeitos $73,4 \%$ obtiveram score maior que 50 em Dor; $78,9 \%$ score maior que 50 na Vitalidade; $77 \%$ obtiveram score maior que 50 no Aspectos Sociais; $69,7 \%$ score maior que 50 na Limitação dos Aspectos Emocionais; $73,4 \%$ score maior que 50 na Saúde Mental. 
Tabela 3 - Pontuação nas oito dimensões do SF-36 com apresentação de média e desvio-padrão. Limeira/SP, Brasil, 2017.

\begin{tabular}{|c|c|c|c|}
\hline Variável & $\begin{array}{c}\text { Pontuação } \\
<50(\%)\end{array}$ & $\begin{array}{c}\text { Pontuação } \\
>50 \text { (\%) }\end{array}$ & Média (DP) \\
\hline \multicolumn{4}{|l|}{ Componente Saúde Física (CSF) } \\
\hline Capacidade Funcional & 48 & 52 & $49,3( \pm 29,35)$ \\
\hline Limitação dos Aspectos Físicos & 60 & 40 & $553( \pm 44,59)$ \\
\hline Dor & 27 & 73 & $69,73( \pm 31,52)$ \\
\hline Estado Geral de Saúde & 49 & 51 & $47,63( \pm 17,04)$ \\
\hline \multicolumn{4}{|l|}{ Componente Saúde Mental (CSM) } \\
\hline Vitalidade & 21 & 79 & $61,24( \pm 19,98)$ \\
\hline Aspectos Sociais & 23 & 77 & $69,4( \pm 27,69)$ \\
\hline Limitação dos Aspectos Emocionais & 30 & 70 & $70,8( \pm 42,44)$ \\
\hline Saúde Mental & 27 & 73 & $62,5( \pm 19,58)$ \\
\hline
\end{tabular}

Fonte: FERNANDES D, et al., 2020.

Na Tabela 4 é possível verificar a condição clínica dos pacientes que obtiveram valor de albumina $\leq 3,0$ gr/dL apresentaram comprometimento em diversas domínios do SF-36, como EGS (72\%), CF (63\%), AF (63\%), SM (54\%), AS (54\%), DF (54\%), AE (54\%). Quanto ao clearance de uréia normalizado (Kt/V) os pacientes com Kt/V $\leq 1,2$ apresentaram comprometimento nos domínios de AF (61\%), SG (55\%), CF (44\%).

Os pacientes que obtiveram valores de $\mathrm{Hb} \leq 10 \mathrm{~g} / \mathrm{dL}$, apresentaram comprometimento na qualidade de vida nos domínios CF, AF, SG, AE, SM todos com 58\%. Com relação a faixa etária dos pacientes em hemodiálise, aqueles com idade entre 65 e 74 apresentaram maior comprometimento na capacidade funcional (60\%), como também, os indivíduos com mais de 75 anos obtiveram resultados abaixo do percentil 50 em vários domínios do SF36 (CF,EGS,EF). A qualidade de vida e tempo de HD mostrou resultados não satisfatórios em quase todos domínios do SF-36 (CF, AF, SG, DF, AE, SM), em pacientes com mais de 10 anos de tratamento.

Tabela 4 - Pontuação<50 nas dimensões do SF 36 e aspectos relacionados à diálise: Albumina $\leq 3 \mathrm{mg} / \mathrm{dL}$; $\mathrm{Kt} / \mathrm{V} \leq 1,2 ; \mathrm{Hb} \leq 10 \mathrm{~g} / \mathrm{dL}$; Idade 65 a 74 anos; HD em anos (11 a 15); CDL e FAV/Permcath. Limeira/SP, Brasil, 2017.

\section{Pontuação <50 (\%)}

\begin{tabular}{|c|c|c|c|c|c|c|c|}
\hline Variável & $\begin{array}{l}\text { Albumina } \\
\leq 3 \mathrm{mg} / \mathrm{dL}\end{array}$ & $\mathrm{Kt} / \mathrm{V} \leq 1,2$ & $\begin{array}{c}\mathrm{Hb} \leq 10 \\
\mathrm{~g} / \mathrm{dL}\end{array}$ & $\begin{array}{l}\text { Idade } \\
65 \text { a } 74\end{array}$ & $\begin{array}{c}\text { HD em } \\
\text { anos } \\
(11 \text { a 15) }\end{array}$ & CDL & $\begin{array}{c}\text { FAV/ } \\
\text { Premcath }\end{array}$ \\
\hline \multicolumn{8}{|l|}{$\begin{array}{l}\text { Componente Saúde } \\
\text { Física (CSF) }\end{array}$} \\
\hline $\begin{array}{l}\text { Capacidade Funcional } \\
\text { (CF) }\end{array}$ & $63 \%$ & $44 \%$ & $58 \%$ & $47 \%$ & $67 \%$ & $67 \%$ & $75 \%$ \\
\hline $\begin{array}{c}\text { Limitação dos Aspectos } \\
\text { Físicos (AF) }\end{array}$ & $63 \%$ & $61 \%$ & $58 \%$ & - & $67 \%$ & $67 \%$ & $62 \%$ \\
\hline Dor & $54 \%$ & - & - & - & $67 \%$ & - & $62 \%$ \\
\hline $\begin{array}{c}\text { Estado Geral de Saúde } \\
\text { (SG) }\end{array}$ & $72 \%$ & $55 \%$ & $58 \%$ & $60 \%$ & $100 \%$ & $83 \%$ & $62 \%$ \\
\hline \multicolumn{8}{|l|}{$\begin{array}{c}\text { Componente Saúde } \\
\text { Mental (CSM) }\end{array}$} \\
\hline Vitalidade & - & - & - & - & - & $50 \%$ & - \\
\hline Aspectos Sociais (AS) & $54 \%$ & - & - & - & - & $83 \%$ & - \\
\hline $\begin{array}{c}\text { Limitação dos Aspectos } \\
\text { Emocionais (AE) }\end{array}$ & $54 \%$ & - & $58 \%$ & - & $67 \%$ & $83 \%$ & $62 \%$ \\
\hline Saúde Mental (SM) & $54 \%$ & - & $58 \%$ & - & $67 \%$ & $50 \%$ & - \\
\hline
\end{tabular}

Fonte: FERNANDES D, et al., 2020.

Os pacientes que utilizavam CDL, apresentaram escores menores de 50 em todos os domínios avaliados pelo questionário SF-36. Em seguida, os portadores de FAV/Permcath apresentaram baixa pontuação em 5 domínios, sendo eles CF, AF, DF, SG, AE. 


\section{DISCUSSÃO}

O desenvolvimento desta pesquisa possibilitou mensurar a qualidade de vida de portadores de DRC, que estavam em hemodiálise, e avaliou a influência de diferentes variáveis sociais, demográficas, econômicas e clínicas sobre a qualidade de vida desses.

$\mathrm{Na}$ caracterização da amostra, foi observada uma maior prevalência da doença em pacientes com idade média de 57 anos e do sexo masculino. Esses dados se assemelham a outros estudos que mostram a prevalencia de homens com DRC em tratamento dialitico com idade superior a 50 anos (GONÇALVES FA, et al., 2015).

Quanto ao estado civil, assim como nos achados de Guerra-Guerrero V, et al. (2012) a maioria dos pacientes em hemodiálise tinham companheiro e viviam com a família. A ausência de um companheiro para um paciente com doença renal crônica pode ser prejudicial para os cuidados com a saúde, além da pior percepção da qualidade de vida.

A escolaridade encontrada nesta pesquisa corrobora junto a demais literaturas acerca do perfil dos pacientes em hemodiálise. O grau de escolaridade e acesso à informação pode contribuir para limitadas ações de autocuidado e prevenção de doenças. É considerada uma variável de grande destaque na caracterização da amostra de portadores de DRC, pois, pode contribuir para avaliar a qualidade das informações prestadas e o nível de compreensão dos pacientes (RIBEIRO WA e ANDRADE M, 2018).

Com relação aos dados econômicos, foi observado maior prevalencia daqueles com renda mensal menor que três salários mínimo. O estudo de Lima AB (2016); Identificou que aproximadamente $43 \%$ dos pacientes estavam afastados da atividade laboral por motivo de saúde e recebendo auxílio-doença do Instituto Nacional do Seguro Social.

Nessa perspectiva, a diferença entre as rendas anteriores à presença da DRC pode ser expressa em virtude do comprometimento das atividades do trabalho, devido às limitações e ao tempo dispendido para realizar a hemodiálise. A baixa renda é um dado que pode ser interpretado como um fator negativo na prevenção de doenças crônicas, uma vez que são necessários recursos e utilização dos serviços de saúde suplemtares, a fim de obter medidas de diagnóstico e tratamento.

Quanto ao plano de saúde utilizado, foi possivel observar que dos pacientes entrevistados, cerca de metade possuem plano de saúde privado. Tal dado vai de encontro à demais estudos que demonstram que os individuos que utilizam o plano de saúde privado buscam maior acessibilidade a procedimentos que viabilizam um diagnostico mais rápido, assim como são direcionados aos tratamentos necessários (MALTA DC, et al., 2017). No entanto, a realidade brasileira, segundo o Censo Brasileiro de Nefrologia, no ano de 2019, $79 \%$ dos pacientes em diálise tiveram como fonte pagadora do tratamento dialítico, o Sistema Único de Saúde (SBN, 2020).

Em relação aos fatores de risco à doença renal, a população teve incidência significativa de diabettes mellitus e hipertensão arterial sistêmica como doença de base. A hipertensão arterial sengundo censo brasileiro de nefrologia é a maior causa de DRC (SBN, 2020). As duas comorbidades são os principais fatores de risco para doença renal crônica, sendo afirmada categoricamente como maior prevalência nos pacientes com DRC (MILAGRES CS, et al., 2015).

Segundo as diretrizes do manejo clinico ao doente renal do Ministério da Saúde, o portador de DRC deve ser acompanhado pela equipe de saúde por meio do tratamento conservador na atenção básica até certo estágio da doença (BRASIL, 2014). Após o estágio 3b, a diretriz determina que o paciente seja direcionado para o tratamento ambulatorial com a especialidade de nefrologia, além da equipe multidisciplinar. Assim, este trabalho corrobora junto à no qual a maioria dos pacientes que iniciam o tratamento dialítico não passaram pelo tratamento conservador, ou seja, desconheciam o risco de doença renal, os fatores agravantes e o tratamento adequado (BASTOS MG e KINZTAJN GM, 2011).

A avaliação das dimensões da qualidade de vida dos pacientes estudados apresentou de forma geral um grau de comprometimento em alguma delas, seja física ou mental, podendo estar relacionada diretamente à condição clínica, emocional ou social, como apresentados também em outros estudos (JESUS NM, et al., 2019; ZANESCO C, et al., 2019). 
Assim como observados em outros estudos, a instabilidade clínica da doença renal é marcante no início do tratamento. Uma vez alcançado um equilíbrio hidroeletrolítico ou uma maior adaptação ao quadro da doença e da terapia dialítica, se percebe melhora na qualidade de vida, pela melhora do equilíbrio, controle das doenças de base, e dos indicadores clínicos (CARNEIRO EB, 2019).

Logo, na estratificação e análise dos dados foi possível verificar que condições clinicas específicas, como albulminúria, impactam diretamente a qualidade de vida no componente de saúde física, sendo condições que estão presentes nos indivíduos que iniciaram o tratamento hemodialitico com diagnóstico tardio da doença renal.

A albumina reduzida, pode estar relacionada ao pior estado nutricional do paciente. No entanto, faz-se necessário relatar que este método bioquímico, amplamente utilizado devido à facilidade de sua medição e associação com efeitos clínicos, pode ser significativamente alterado pela DRC e nem sempre reflete o estado nutricional do paciente, podendo também ser modificado por perdas pelo dialisato (solução de diálise), doenças sistêmicas, idade avançada e presença de inflamação (CUPPARI L e KAMIMURA MA, 2009).

Inesperadamente, foi verificado que a variável tempo em tratamento hemodialítico de 11 a 15 anos, apresentou o maior score para o domínio de estado geral da saúde. Situação contrária a demais trabalhos, nos quais o tempo em tratamento hemodiálitico está relacionado a melhores pontuações nos domínios físicos e domínios relacionados aos aspectos sociais e emocionais. Estes dois ultimos, também estiveram presentes em $67 \%$ dos indivíduos que apresentaram pontuação menor que 50 pelo SF-36.

Referente à influência no domínio físico, as pesquisas tem levantado a hipótese de que indivíduos que realizam o tratamento hemodialítco a maior tempo têm significativa diminuição das escórias nitrogenadas, maior redução dos sintomas de uremia, contribuindo para a melhora física e consequentemente, refletindo nas condições emocionais no desempenho das atividades diversas, assim cmo proporcioando melhor percepção de bem-estar (BERNARDO MF, et al., 2019; JESUS NM, et al., 2019).

Vale ressaltar que a HD provoca diversos tipos de limitações nos indivíduos submetidos a este tratamento de alta complexidade. As limitações físicas e mentais com o progredir do tempo de tratamento podem reduzir as complicações hidroeletrolíticas, a percepção do medo, contribuindo para enfrentamento da doença, das restrições impostas pela mesma, assim como para melhora da percepção da saúde geral.

Cavalcante MCV, et al. (2013) puderam verificar que influência do tempo de tratamento na melhor QV da qualidade vida quando os portadores de DRC comparavam sua saúde a tempos anteriores, corroborando aos demais estudos já sugeridos.

A utilização de acesso vasculares central de curta permanência (CDL) são mais prevalentes em situações de urgência dialítica na ou impossibiliade da utilização ou confecção de um acesso vascular definitivo (MARINHO CLA, et al, 2017). Neste último, a utilização do permcath faz-se necessário. Observa-se nestes dois tipos de acessos que os riscos de infecção pelo processo invasivo, assim como uma baixa qualidade de diálise podem contribuir para que estes pacientes apresentem comprometimento em vários domínios do SF36, tanto nos aspectos físicos quanto mentais, devido ao processo de deterioramento clinico e aos procedimentos invasivos (CARNEIRO EB, 2019).

Estes achados puderam ser observados no presente estudo, no qual a utilização de CDL em indivíduos em tratamento hemodilítco apresentou maiores propoções de comprometimento no estado geral de saúde, assim como nos aspectos sociais e emocionais, refletindo em uma pior QV na percepção da condição de saúde física e emocional para o desempenho das atividades (MARINHO CLA, et al, 2017; PEREIRA CV e LEITE IC, 2019).

A utilização de FAV/Permcath no desempenho das atividades diárias no compomente de saúde física apresentou maior percentual de pontuação<50 nos dados dialíticos. Assim como a utilização de CDL, o Permcath contribui para maior risco de de infecção pelo processo invasivo, assim como pode ser verificado em pacientes com impossibilidade de confesção de FAV (PEREIRA CV e LEITE IC, 2019). 
Com relação às limitações do estudo, destaca-se que, embora contemple pacientes renais em tratamento hemodialítico, foi realizado em apenas um município, o que implica que as generalizações devem ser realizadas com cautela. No mais, o delineamento transversal desta pesquisa não é capaz de permitir demais relações entre causa e efeito entre a qualidade de vida e os diversos fatores analisados no estudo.

\section{CONCLUSÃO}

Concluímos que a DRC causa impacto nos diferentes domínios dos compenentes físico e mental nos pacientes em tratamento hemodialítico. Tais comprometimentos podem implicar em significativo impacto, desempenho e percepção destes indivíduos sobre suas QV frente à doença e seu tratamento, um acompanhamento e monitoramento da equipe multidisciplinar. O instrumento utilizado é considerado uma excelente forma de mensurar a avaliação da qualidade de vida e suas informações bem delineadas podem contribuir para a construção de um projeto terapêutico individualizado à necessidades de cada paciente, buscando, portanto, contribuir para melhora na qualidade de vida, além de ser um diferencial para as instituições que buscam a qualidade em saúde e a excelência no atendimento prestado.

\section{REFERÊNCIAS}

1. BASTOS MG, KINZTAJN GM. Doença renal crônica: importância do diagnóstico precoce, encaminhamento imediato e abordagem interdisciplinar estruturada para melhora do desfecho em pacientes ainda não submetidos à diálise. J. Bras. Nefrol. 2011; 33(1):93-108.

2. BERNARDO MF, et al. Estado nutricional e qualidade de vida de pacientes em hemodiálise. Medicina (Ribeirão Preto Online). 2019; 52(2):128-35.

3. BRASIL. Ministério da Saúde. Secretaria de Atenção à Saúde. Departamento de Atenção Especializada e Temática. Diretrizes Clínicas para o Cuidado ao paciente com Doença Renal Crônica - DRC no Sistema Único de Saúde/ Ministério da Saúde. Secretaria de Atenção à Saúde. Departamento de Atenção Especializada e Temática. - Brasília: Ministério da Saúde, 2014. p.: 37.

4. CARNEIRO EB. Associação da qualidade de vida dos nefropatas crônicos com o tipo de acesso vascular para hemodiálise. Dissertação (Mestrado em Cirurgia) - Programa de Pós-Graduação em Cirurgia. Universidade Federal do Amazonas, Manaus, 2019; 79 p.

5. CAVALCANTE MCV, et al. Factors associated with the quality of life of adults subjected to hemodialysis in a city in northeast Brazil. J Bras Nefrol. 2013; 35(2):79-86.

6. CICONELLI RM. Tradução para o português e validação do Questionário genérico de avaliação de qualidade de vida "Medical outcomes study 36-item short-form helth survey (SF-36)". Tese (Doutorado em Medicina). Universidade Federal de São Paulo (UNIFESP). São Paulo (SP),1997; 120p.

7. CUPPARI L, KAMIMURA MA. Avaliação Nutricional na Doença Renal Crônica: desafios na prática cllínica. J Bras Nefrol. 2009; 31(supl.1): 28-35.

8. DIAS EC, et al. Avaliação dos índices de infecção relacionados ao cateter duplo lúmen para hemodiálise antes e após orientação para o autocuidado. Revista Uningá, Maringá, 2017; 53 (2): 18-25.

9. GUERRA-GUERRERO V, et al. Qualidade de vida de pessoas em hemodiálise crônica: relação com variáveis sociodemográficas, médico-clínicas e de laboratório. Rev Latino-Am Enfermagem 2012; 20 (5):838-46.

10. GONÇALVES FA, et al. Qualidade de vida de pacientes renais crônicos em hemodiálise ou diálise peritoneal: estudo comparativo em um serviço de referência de Curitiba- PR. Jornal Bras. Nefrol., 2015; 37(4): 467-474.

11. JESUS NM, et al. Qualidade de vida de indivíduos com doença renal crônica em tratamento dialítico. J. Bras. Nefrol., São Paulo, 2019;41(3):364-374.

12. LIMA AB. Estresse, depressão e suporte familiar em pacientes em diálise peritoneal e hemodiálise. Dissertação (Mestrado em Psicologia do Desenvolvimento e Aprendizagem) - Faculdade de Ciências - Campus Bauru. Universidade Estadual Paulista Júlio de Mesquita Filho (UNESP), Bauru-SP, 2016; 73p.

13. MALTA DC, et al. Doenças crônicas não transmissíveis e a utilização de serviços de saúde: análise da Pesquisa Nacional de Saúde no Brasil. Rev Saude Publica. 2017; 51 Supl 1:4s.

14. MARINHO CLA, et al. Qualidade de vida de pessoas com doença renal crônica em hemodiálise. Rev Rene 2017; 18:396-403.

15. MILAGRES, CS et al. Prevalência e fatores associados à presença de anemia em idosos do município de Viçosa (MG), Brasil. Ciênc. saúde coletiva, Rio de Janeiro, 2015; 20(12): 3733-3741.

16. NEVES PDMM, et al. Brazilian Dialysis Census: analysis of data from the 2009-2018 decade. Braz. J. Nephrol. 2020;42(2):191-200.

17. PEREIRA CV, LEITE IC. Qualidade de vida relacionada à saúde de pacientes em terapêutica hemodialítica. Acta Paul Enferm. 2019; 32(3): 267- 74 .

18. RIBEIRO WA, ANDRADE M. Enfermeiro protagonista na educação em saúde para o autocuidado de pacientes com doenças renal crônica. Revista Pró-univerSUS. 2018; 9(2): 60-65.

19. SOCIEDADE BRASILEIRA DE NEFROLOGIA. Relatório do censo brasileiro de diálise. Jornal Brasileiro de Nefrologia, São Paulo, 2020; 42(2):191-200.

20. THOME FS, et al. Brazilian chronic dialysis survey 2019. Braz. J. Nephrol. 2019;41(2):208-214.

21. ZANESCO C, et al. Avaliação da qualidade de vida de pacientes renais crônicos em hemodiálise: um estudo transversal. Revista de Pesquisa: Cuidado e Fundamental. 2019; 11(1): 186-91. 